\title{
High Optical Efficiency of ZnO Nanoparticles
}

\author{
Sumeyra Tek ${ }^{\mathrm{a}, \mathrm{c}}$ and Hilmi Volkan Demir ${ }^{\text {a.b.c }}$ \\ ${ }^{a}$ Department of Physics, ${ }^{b}$ Department of Electrical and Electronics Engineering, \\ ${ }^{c}$ Nanotechnology Research Center, \\ Bilkent University, Ankara, 06800, Turkey \\ Dilek Yucel and Gulsen Celiker \\ DYO, Yasar Group, A. O. S. B., 10003 Sk. No:2, Cigli, Izmir 35620 Turkey \\ volkan@bilkent.edu.tr
}

\begin{abstract}
We develop optically efficient photocatalytic $\mathrm{ZnO}$ nanoparticles that we chemically embed and well disperse into host PVAc thin films and experimentally demonstrate the highest optical efficiency of $\sim 70 \%$ in $\mathrm{ZnO}$ nanoparticle films, with increasing optical spectral efficiency as the excitation wavelength is swept from $370 \mathrm{~nm}$ to $290 \mathrm{~nm}$.

(C) 2007 Optical Society of America

OCIS codes: Nanophotonics, (160.4670) Optical materials, (160.4760) Optical properties
\end{abstract}

Nanostructured metal-oxide semiconductors find interesting applications in nanophotonics [1-5]. They exhibit peculiar optoelectronic and photocatalytic properties that make them attractive, for example, for optical decontamination of surfaces, air, and water. For such environmental purposes, we develop photocatalytic $\mathrm{ZnO}$ nanoparticles integrated in resin and study their optical spectral efficiency between 290 $\mathrm{nm}$ and $370 \mathrm{~nm}$. We experimentally achieve very high optical efficiencies up to $70 \%$ in $\mathrm{ZnO}$ nanoparticle films for the first time.

In photocatalytic processes, semiconductors such as $\mathrm{TiO}_{2}, \mathrm{ZnO}, \mathrm{CdS}$, and $\mathrm{ZnS}$ are optically activated in ultraviolet (UV) radiation $[1,2] . \mathrm{TiO}_{2}$ in the anatase form is the most common one. However, $\mathrm{ZnO}(3.2 \mathrm{eV})$ is a better alternative to $\mathrm{TiO}_{2}$ as far as its bandgap energy is concerned, although it is less commonly used. $\mathrm{ZnO}$ is the last member of the $3 \mathrm{~d}$ metal-oxide series, with $\mathrm{Zn}$ completely filling its $3 \mathrm{~d}$ orbitals $\left(3 \mathrm{~d}^{10}\right)$. $\mathrm{ZnO}$ 's conduction band has s-p hybridized orbitals while its valence band has only $d$-states. This results in the dissimilar parity of the photoexcited $\mathrm{e}^{-}-\mathrm{h}^{+}$pair in $\mathrm{ZnO}$, leading to a low recombination probability, as in $\mathrm{TiO}_{2}$. Thus, $\mathrm{ZnO}$ and $\mathrm{TiO}_{2}$ are the only two among the $3 \mathrm{~d}$ transition metal-oxide semiconductor series that remain stable upon photoexcitation [3]. It is also reported in the literature that $\mathrm{ZnO}$ powder has significantly larger quantum efficiency than that of $\mathrm{TiO}_{2}$ powder [1]. This motivates us to investigate optical efficiency characteristics of $\mathrm{ZnO}$.

To this end, many research studies about $\mathrm{ZnO}$ in aqueous solutions have been conducted. However, little research is available for $\mathrm{ZnO}$ and its photocatalytic efficiencies when $\mathrm{ZnO}$ nanparticles are embedded and immobilized in thin films (although immobilized form is comparatively more versatile in industrial applications). In this work, we thus work on the optical efficiency characterization of $\mathrm{ZnO}$ nanoparticles integrated in resin and report on very high spectral efficiencies for such $\mathrm{ZnO}$ nanoparticle films for the first time.

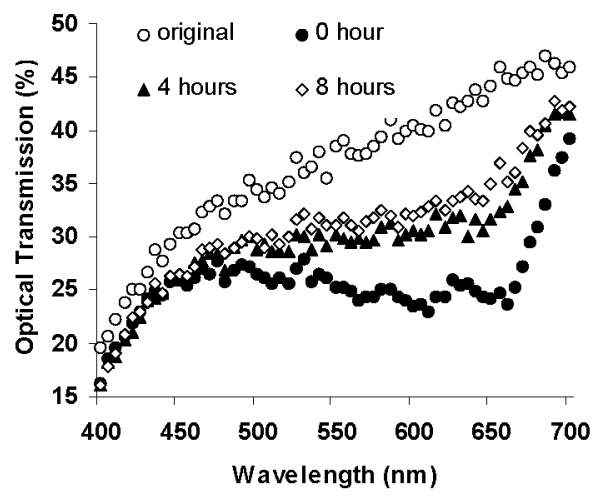

Fig. 1. Optical transmission spectra of our zinc oxide nanoparticles film right before (original) and after ( 0 hour) it is contaminated with methylene blue, and subsequently when optically activated for 4 and 8 hours at $290 \mathrm{~nm}$. With optical activation in UV, the initial contamination is recovered by $70 \%$. 
We use $\mathrm{ZnO}$ particles of $100 \mathrm{~nm}$ in size. We chemically integrate and disperse these $\mathrm{ZnO}$ nanoparticles into host PVAc (poly(vinylacetate)/ poly(methylmethacrylate)) resin. We form thin films of the resulting $\mathrm{ZnO}$ nanocomposite by spraying. We contaminate our samples with the standard metylene blue for optical characterization. We optically activate at certain wavelengths in the UV (from $370 \mathrm{~nm}$ to $290 \mathrm{~nm}$ ) and perform optical transmission spectroscopy in the visible (between $450 \mathrm{~nm}$ and $700 \mathrm{~nm}$ ). During the optical activation process, we observe visual changes in the color of our samples. Figure 1 shows typical optical transmission spectra through our $\mathrm{ZnO}$ nanoparticle film with no contamination (original) and with the contamination but no optical activation ( 0 hour). Due to the contamination, there is a significant drop in the optical transmission. However, with the optical activation (for 4 hours and 8 hours with an optical power of $120 \mu \mathrm{W}$ at a wavelength of $290 \mathrm{~nm}$ ), the $\mathrm{ZnO}$ nanoparticle film recovers its optical transmission back up by $70 \%$ at the end of 8 hours. This optical recovery is attributed mostly to the photocatalytic activity of $\mathrm{ZnO}$ nanoparticiples. The degradation of the host resin may also in part contribute to the optical recovery of the transmission.

For a complete study of optical spectral efficiency characterization, we study the changes in the optical transmission of $\mathrm{ZnO}$ nanoparticles in the visible spectra as we change the excitation wavelength in the UV. We use a measure of optical efficiency to evaluate and compare the photocatalytic activity under UV exposure at different wavelengths. We extract the optical efficiency from the spectral area between the optical transmission curves of the sample before and after optical activation normalized with respect to the spectral area between the transmission curves before and after contamination across a wide spectral region (in the visible between $450 \mathrm{~nm}$ and $700 \mathrm{~nm}$ ), over which the effect of the contamination and decontamination is observed. Figure 2 shows the optical efficiencies of our $\mathrm{ZnO}$ nanoparticles for excitation wavelengths between $290 \mathrm{~nm}$ and $370 \mathrm{~nm}$ as a function of incident total optical energy per unit area (between 1.8 Joules $/ \mathrm{cm}^{2}$ and 7.6 Joules $/ \mathrm{cm}^{2}$ ). We demonstrate that optical efficiencies increase with decreasing excitation wavelengths. We obtain high optical efficiency up to $70 \%$ at $290 \mathrm{~nm}$ shown in Fig. 2.

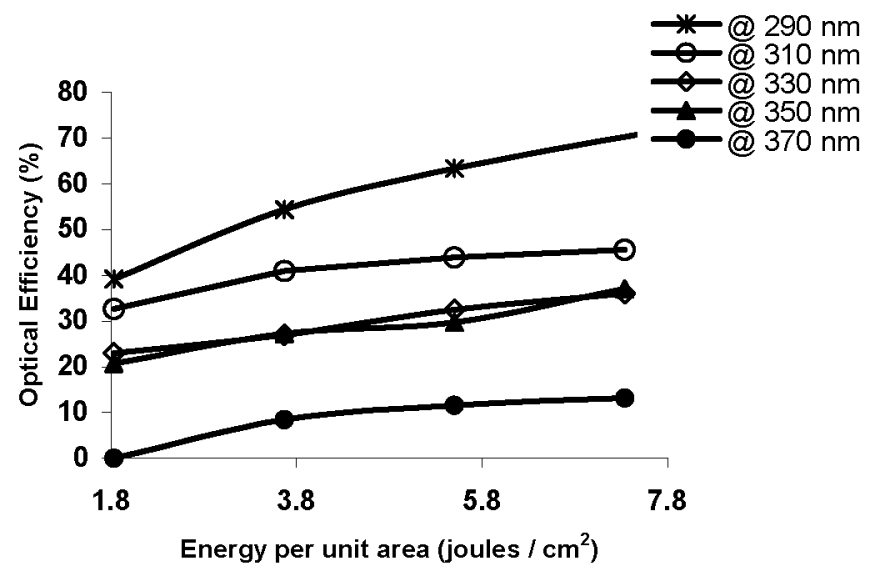

Fig. 2. Optical efficiency of our zinc oxide nanoparticles at different activation wavelengths between $290 \mathrm{~nm}$ and $370 \mathrm{~nm}$ as a function of incident total optical energies per unit area.

In conclusion, we developed and investigated photocatalytic $\mathrm{ZnO}$ nanoparticles integrated in thin films for optically induced decontamination and characterized their optical spectral efficiency at excitation wavelengths from $290 \mathrm{~nm}$ to $370 \mathrm{~nm}$. We experimentally demonstrated that optical efficiencies increase with decreasing excitation wavelength. We obtained the highest optical efficiency of $70 \%$ at $290 \mathrm{~nm}$. We are currently working on the development of $\mathrm{ZnO}$ nanoparticles embedded in other resins to further study and identify optically induced degradation mechanisms.

\section{References}

[1] S. Chakrabarti, B. K. Dutta, "Photocatalytic degradation of model textile dyes in waste water using ZnO as semiconductor catalyst," Journal of Hazardous Materials 112, 269-278 (2004)

[2] G. Mascolo, R. Comparelli, M.L. Curri, G. Lovecchio, A. Lopez \& A. Agostiano, "Photocatalytic degradation of methylred by

$\mathrm{TiO}_{2}$ : Comparison of the efficiency of immobilized nanoparticles versus conventional suspended catalyst," Journal of Hazardous Materials (2006) (in press)

[3] S. Banerjee, J. Gopal, P. Muraleedharan, A.K. Tyagi \& B. Raj, "Physics and chemistry of photocatalytic titanium dioxide: visualization of bactericidal activity using atomic force microscopy," Current Science 90, 1378-1383 (2006).

[4] S. Kundu, S. K. Ghosh, M. Mandal, T. Pal \& A. Pal, "Spectrophotometric determination of arsenic via arsine generation and in-situ color bleaching of methylene blue (MB) in micellar medium," Talanta 58, 935-942 (2002). 